\title{
Why a Special Issue on Gene Therapy?
}

\author{
Nicol Keith ${ }^{1}$ and Claude Bagnis ${ }^{2}$ \\ ${ }^{1}$ Cancer Research UK Department of Medical Oncology, University of Glasgow, UK \\ ${ }^{2}$ Department of Cellular and Gene Therapy, EFS Alpes Méditerranée, 13009 Marseille, Cedex 5, France
}

Going both from the organism to the DNA and from the DNA to the organism, the biology of the 20th century has paved the way for a new type of biologist who has emerged from the laboratory during the last two decades: the gene therapist. At the beginning of the nineties, much of this young field was based on a relatively simple concept: a deficient function can be replaced with an artificial gene. For twenty years now, gene therapists have been learning how wide the gap is between a concept and its practical reality. However, according to the increasing numbers of papers published which relate to gene therapy, and the huge interest generated by meetings of the American or the European Society of Gene Therapy, we are certainly learning a lot.

So, what have we learnt? Firstly, biotechnology is the key to link the bench to the bed of the patient. The biotechnology industry is ideally placed to deal with the concept of biotherapeutics and the range of manufacturing issues including good manufacturing practice (GMP), safety, toxicology, product scale-up to generate large batches of vectors, and testing new gene transfer tools. The close contacts between biotech companies and the development of gene therapy highlight the technological aspects of gene therapy. Indeed, delivery of the gene therapy has emerged as essential area for improvement. Gene delivery is a major growth industry with approaches ranging between almost-naked DNA to miniviruses and viral vectors gutted of most if not all of the viral sequences of their original genome. In addition, there has been increased and imaginative use of unexpected viruses as gene transfer agents, as highlighted by N. Maitland et al in their review of baculovirus to transfer therapeutic genes. Where is the universal vector everybody was running after 10 years ago?

We have also learned that between the needle and the arm of the patient there is a world called "Biosafety." There is no "zero risk" when considering a biotherapeutical strategy. In addition, gene therapy is a complex biological issue which generates specific risks as recently evidenced during the treatment of genetic diseases with adenoviral and retroviral vectors. It can be suggested that gene therapy is not toxic because gene therapy is not efficient. This opinion may hold some truth, but the use of increasing doses, the use of more complex strategies, and the use of more potent genetic activities is now leading us to reach the borderline between safety and toxicity making the biosafety a major determinant in the future of this therapeutical approach. Biosafety has to consider both the design of a gene transfer product and the possibility for this product to bypass the safety keys set up by the investigator to avoid the induction of unexpected deleterious effects. In this respect, in this two-part special issue T. Robson and D. G. Hirst (part II) focus on transcriptional targeting and A. G. Schätzlein (part II) focuses on the targeting of synthetic gene delivery. These reviews discuss one of the most important aspects of biosafety: the possibility to restrict the expression of the gene of interest to the selected tissue. In addition, A. Van den Broeke and A. Burny (part I) remind us that gene transfer vectors have to be considered in appropriate animal models by taking advantage of an elegant sheep model that allow us to revisit the concept of retroviral vector stability and recombination potential.

We learned that there is not always a simple solution. Cardiovascular disease is a complex multiparameter disorder. However, innovative biological therapies as discussed by K. L. Dishart et al (part II) highlight some very exciting advances in the field of cardiovascular disease. It will be of interest over the next few years to analyze whether clinical results will reach the expectations in this domain bearing in mind the lessons of gene therapy for cancer. The treatment of cancer, makes up almost 60 to $70 \%$ of the gene therapy trials worldwide, and includes some very elegant approaches (reviewed by D. H. Palmer et al and by S. M. Scholl et al (part I)). Nevertheless, gene therapy for cancer remains a challenging issue to address despite 15 years of effort and experience as exemplified by the gene therapy of malignant glioma (T. Kanzawa et al (part I)) and pediatric cancer (E. Biagi et al (part I)). Improvements however, can be reassured through our understanding of tumour biology and advances in chemistry. Examples of such improvements involve the synergy 
between therapeutical strategies (R. J. Mairs and M. Boyd (part II)), the use of cascade mechanisms such as strong bystander effects (W. A. Denny (part I)), or the boosting of the immune system (S. M. Scholl et al (part I)). The possibility to target the expression of a gene at the desired time and location as reviewed in this issue will obviously help us to improve the therapeutic impact of these approaches.

Measuring the success of a new therapy is a challenge in itself. The traditional criteria used to evaluate cancer therapies may not be applicable to molecularly driven therapeutics. As we enter the era of mechanismbased therapeutics, it will be essential to show that the new genetic therapies reach their desired target and interacts with the target in a specific fashion. An essential part of this process is therefore the development of new approaches to visualize the localization of the transgene and the extent of its effects. The green fluorescent protein or the bacterial beta-galactosidase encoded by the LacZ gene gave some insight to product localization. But moving into animal models highlights the need for new detection technologies such as high sensitivity detection of light and PET imaging. These advances in noninvasive imaging in vivo are discussed by G. Vassaux and T. Groot-Wassink (part II). This is an emerging field which faces the challenge of detecting and mapping a few if not single cells, in a $3 \mathrm{D}$ context in the next two decades. A nice bet.

Finally, it is encouraging that individual groups worldwide are addressing local requirements to ensure the progress of fledgling genetic therapies into the clinic. However, it is becoming clear that a greater cooperation and unification of regulatory procedures would enhance the prospects of successful clinical trials for gene therapy. Amati et al discuss how this might be achieved within Europe; a major but worthwhile task.

In summary, we learned a lot over the past twenty years. It is a particularly exciting time to be involved in gene therapy. There has been immense progress in the field and a realistic expectation that gene therapy will make a difference to the patient. However, we still have much to learn and this is one of the goals of this two-part special issue on gene therapy of the Journal of Biomedicine and Biotechnology.

Nicol Keith Claude Bagnis

Nicol Keith is the Head of the Telomerase Therapeutics Group at the Cancer Research UK Beatson Laboratories, University of Glasgow. His research interests focus on translational research extending the identification of basic mechanisms of gene regulation into validated targets for new cancer therapeutics including gene therapy, transcriptional modulators, and signal transduction inhibitors.
Claude Bagnis is the Head Scientist of the Department of Cell and Gene Therapy of the Etablissement Français du Sang Alpes Méditerranée in Marseille, France. His projects mainly focus on therapeutic gene transfer in the field of oncology both considering hematopoietic cells or solid tumor cells as targets and retroviral vectors as gene transfer

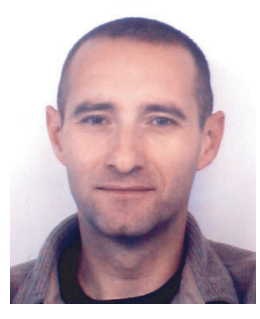
tools. He started his works in 1986 with avian leukemia virusderived vectors in the École Normale Supérieure of Lyon before he joined in 1991 the Centre Régional de Lutte Contre le Cancer in Marseille, where he switched to murine leukemia virusderived retroviral vectors as a tool to genetically modify human hematopoietic stem cells with marker genes in the clinical context. Since 2001, as the Head Scientist of a task group dedicated to the involvement of the Etablissement Français du Sang in the onset of clinical trials using the viral vector technology, Claude Bagnis is mainly developing the use of human immunodeficiency virus-derived vectors to transfer killer genes such as prodrug-dependent suicide genes, proapoptotic genes, or immune response inducers for cancer gene therapy strategies. 

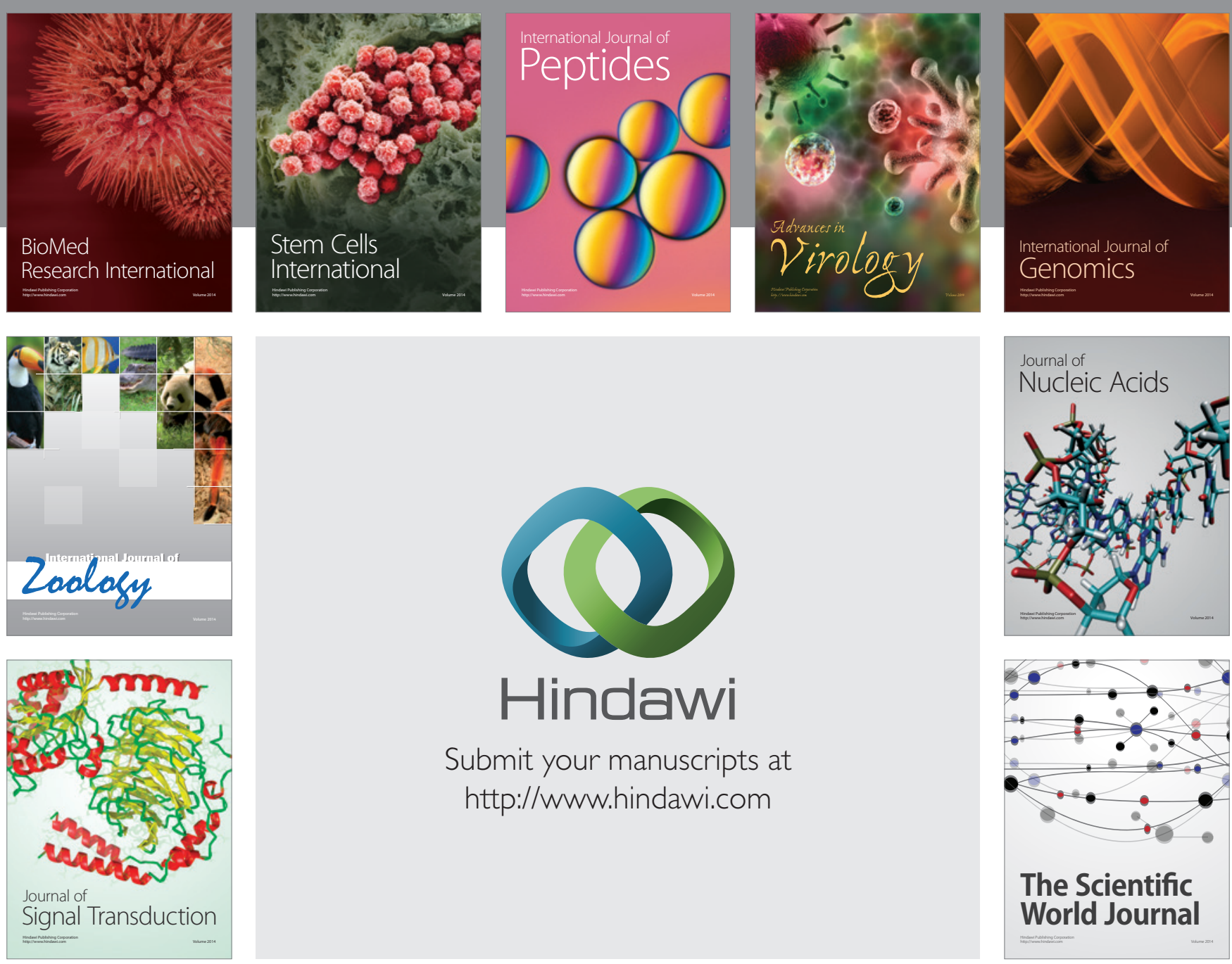

Submit your manuscripts at

http://www.hindawi.com
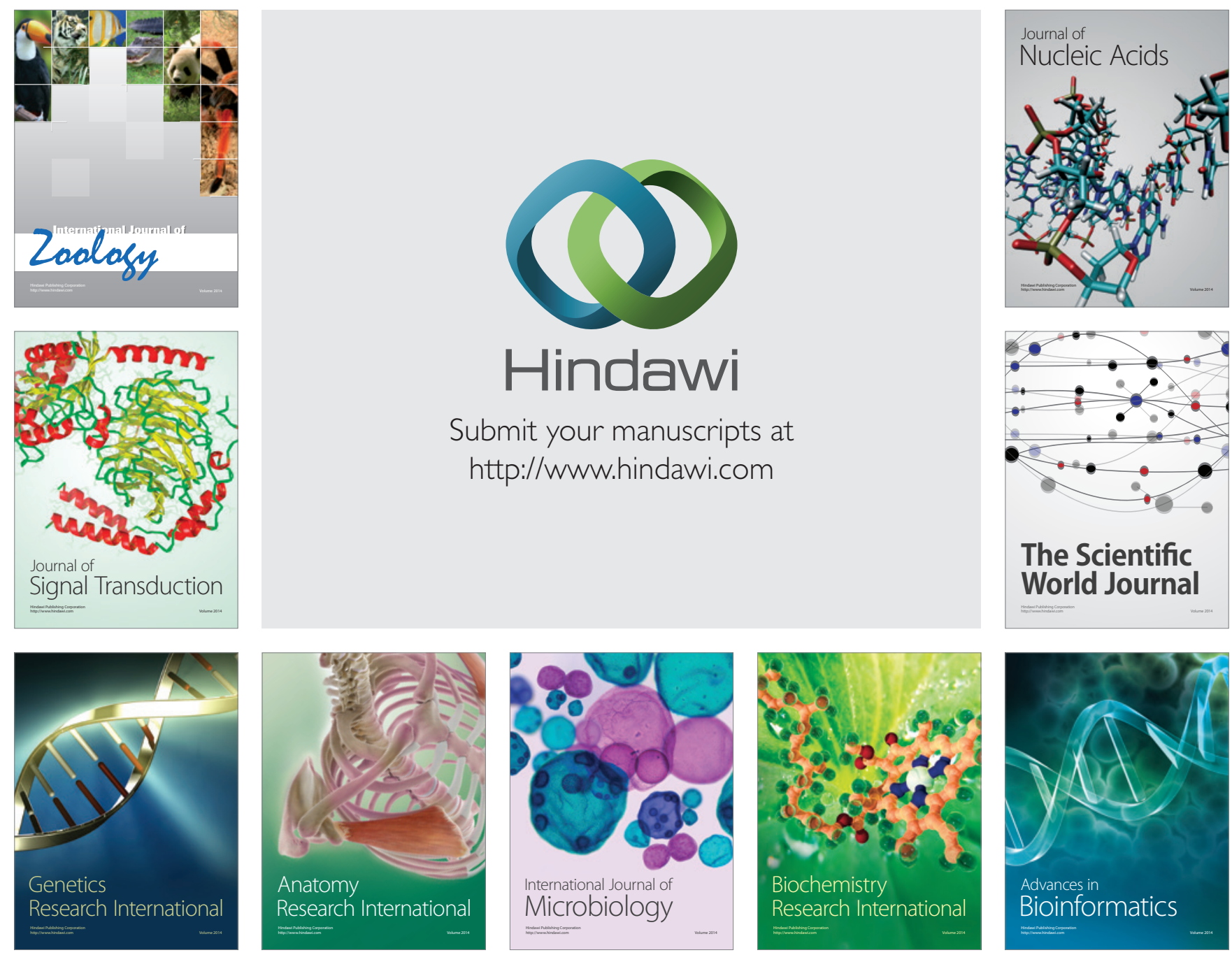

The Scientific World Journal
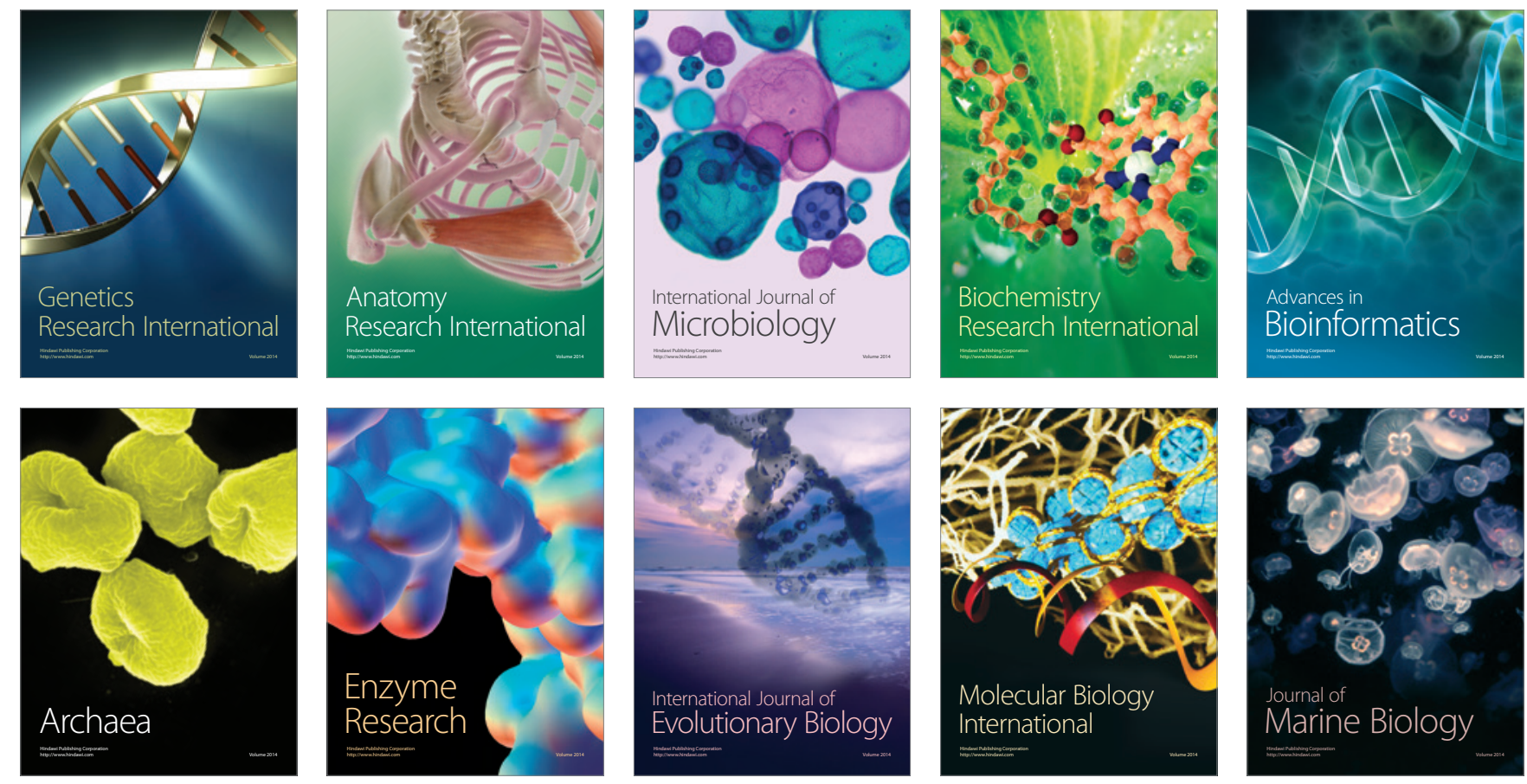